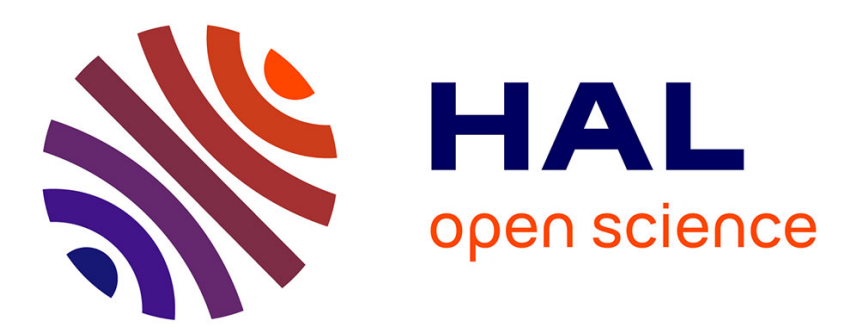

\title{
A minimal dynamical model for tidal synchronization and orbit circularization
}

József Vanyó, Bruno Escribano, Julyan H. E. Cartwright, Diego L. González, Oreste Piro, Tamás Tél

\section{- To cite this version:}

József Vanyó, Bruno Escribano, Julyan H. E. Cartwright, Diego L. González, Oreste Piro, et al.. A minimal dynamical model for tidal synchronization and orbit circularization. Celestial Mechanics and Dynamical Astronomy, 2010, 109 (2), pp.181-200. 10.1007/s10569-010-9322-2 . hal-00595948

\section{HAL Id: hal-00595948 \\ https://hal.science/hal-00595948}

Submitted on 26 May 2011

HAL is a multi-disciplinary open access archive for the deposit and dissemination of scientific research documents, whether they are published or not. The documents may come from teaching and research institutions in France or abroad, or from public or private research centers.
L'archive ouverte pluridisciplinaire HAL, est destinée au dépôt et à la diffusion de documents scientifiques de niveau recherche, publiés ou non, émanant des établissements d'enseignement et de recherche français ou étrangers, des laboratoires publics ou privés. 
Noname manuscript No.

(will be inserted by the editor)

\title{
A minimal dynamical model for tidal synchronization and orbit circularization
}

\author{
József Vanyó $^{1}$. Bruno Escribano ${ }^{2}$. \\ Julyan H. E. Cartwright ${ }^{2}$ Diego L. González ${ }^{3}$ \\ Oreste Piro $^{4}$. Tamás Tél ${ }^{1}$
}

Received: date / Accepted: date

\begin{abstract}
We study tidal synchronization and orbit circularization in a minimal model that takes into account only the essential ingredients of tidal deformation and dissipation in the secondary body. In previous work we introduced the model [7]; here we investigate in depth the complex dynamics that can arise from this simplest model of tidal synchronization and orbit circularization. We model an extended secondary body of mass $m$ by two point masses of mass $m / 2$ connected with a damped spring. This composite body moves in the gravitational field of a primary of mass $M \gg m$ located at the origin. In this simplest case oscillation and rotation of the secondary are assumed to take place in the plane of the Keplerian orbit. The gravitational interactions of both point masses with the primary are taken into account, but that between the point masses is neglected. We perform a Taylor expansion on the exact equations of motion to isolate and identify the different effects of tidal interactions. We compare both sets of equations and study the applicability of the approximations, in the presence of chaos. We introduce the resonance function as a resource to identify resonant states. The approximate equations of motion can account for both synchronization into the $1: 1$ spinorbit resonance and the circularization of the orbit as the only true asymptotic attractors, together with the existence of relatively long-lived metastable orbits with the secondary in $p: q$ ( $p$ and $q$ being co-prime integers) synchronous rotation.
\end{abstract}

Keywords tidal friction $\cdot$ locking $\cdot$ resonance $\cdot$ spin-orbit coupling

\section{Introduction}

Ever since Darwin [4] models have been constructed of the tidal synchronization of the Moon and other celestial bodies [14, 9, 8, 20, 18, 1, 3, 5, 2, 6, 22].

These models have become more quantitatively accurate, but at the same time more complex. Here we take the opposite approach: we strip the problem of tidal synchronization

\footnotetext{
${ }^{1}$ Institute for Theoretical Physics, Eötvös University, P.O. Box 32, H-1518 Budapest, Hungary E-mail: vanyoj@general.elte.hu

${ }^{2}$ Instituto Andaluz de Ciencias de la Tierra, CSIC-Universidad de Granada, E-18071 Granada, Spain

${ }^{4}$ Istituto IMM-CNR, Area della Ricerca CNR di Bologna, I-40129, Bologna, Italy

${ }^{3}$ Departament de Física, Universitat de les Illes Balears, E-07122 Palma de Mallorca, Spain
} 
to its bare bones with a minimal model that captures just the qualitative dynamical aspects of the problem.

To our knowledge there are only two approaches modelling tidal interaction as a fewbody problem. Hut [11] investigates a close binary system where the primary is modelled by three gravitationally interacting point masses (the secondary is point like). Equilibrium tides are described, and the effect of weak friction is taken into account by means of the introduction of a constant time lag. In the approach of Hurford and Greenberg [10] the primary is modelled as a sphere coupled to two point masses via damped springs which do not keep their straight shape but can become curved. The point-like secondary can move along a spherical orbit only and the primary is assumed to spin uniformly.

Although our model has a secondary consisting of two point masses only bound with a damped spring, no specific restrictions are applied. We can thus study dynamical tides, the nonuniform rotation of the secondary, resonances between rotation and orbital motion, and the effect of circularization. This simple model fundamentally differs from others where the secondary is described as a spatially extended object. The two-point-mass feature is nevertheless similar in spirit to restricting those models to the $l=2$ normal mode, as done e.g. in Mardling [15], [16] and Witte et al [24]. It is perhaps due to this feature that we find chaotic dynamics as Mardling [15] did although, owing to the presence of dissipation, in our case chaos is unavoidably of a transient character. A somewhat related problem is that of tethered satellite systems $[13,22]$ but dissipation is typically ignored in this approach.

Our minimal model also enables us to investigate the nature of resonances in detail. We introduce a new definition of resonant states based on a resonance function which distinguishes resonances $p: p$ and $1: 1$, and characterizes the different types of temporal behaviour. A necessary condition for the existence of a resonance is formulated: the energy of the secondary should be constant on average. The response of the dynamics of the secondary to the temporal change of orbital elements can be monitored.

The model is well-defined and easy to treat numerically in its original form [7]. Nevertheless, we carry out a large distance Taylor expansion $(l \ll r)$ over the exact equations of motion. The approximate equations enable us to identify and isolate the different effects of tidal interactions. Special attention is paid to chaotic cases where the applicability of the expansion method is nontrivial. Our finding is, that the exact and approximate equations might lead to different resonances (quasi-attractors) owing to sensitivity to the initial conditions, but if the two solutions reach the same asymptotic state, even the second-order approximation provides a rather accurate description of the dynamics over long times. We can also obtain insight into apapsis precession and the temporal change of orbital elements via the approximate equations.

The paper is organized as follows. The model is introduced in Section 2. The succeeding Section is devoted to a discussion of resonant and chaotic states, and contains the definition of the novel resonance function. The energy condition for resonant states is also given there. The approximate equations of motion are derived, and their application to the energy condition is worked out in Section 4. The change of orbital elements and the comparison of the exact and approximate equations are the subjects of the following two sections. Finally, a discussion follows that also summarizes the results which are expected to be valid beyond the particular model. Technical details are relegated to the Appendix. 

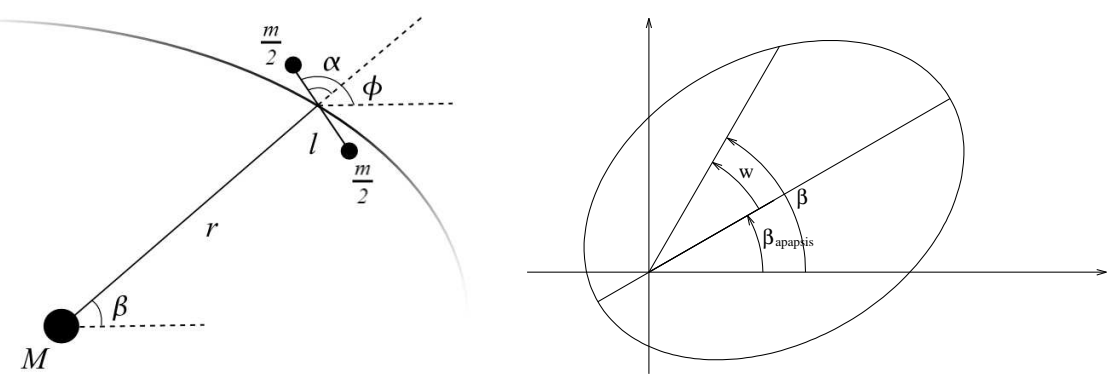

Fig. 1 (a) Instantaneous configuration of the system given by the generalized coordinates $r, \beta, l, \phi$. The relative angle $\alpha=\phi-\beta$ is indicated. (b) Schematic diagram illustrating $\beta_{\text {apapsis }}(t)$

\section{The model}

We model an extended secondary body of mass $m$ by two point masses of mass $m / 2$ connected with a damped spring; see Fig. 1(a). The spring and damper or dashpot are in parallel in a Kelvin-Voigt configuration [17].

This composite body moves in the gravitational field of a primary of mass $M \gg m$ located at the origin. In this simplest case oscillation and rotation of the secondary are assumed to take place in the plane of the Keplerian orbit. We use polar coordinates $r, \beta$ for the centre of mass of the secondary, with $l$ as the instantaneous length of the spring and $\phi$ as the rotational angle characterizing the orientation of the secondary. Both angles $\beta$ and $\phi$ are measured from the $x$-axis in an inertial reference frame. The gravitational interactions of both point masses with the primary are taken into account, but that between the point masses is neglected.

To describe the conservative part of the dynamics we construct a dimensionless Lagrangian, with the initial semi-major axis as the unit of length and the corresponding Keplerian period as the unit of time, in terms of the generalized coordinates $\mathbf{q}=(r, \beta, l, \phi)$ :

$$
\mathscr{L}=\frac{\dot{r}^{2}}{2}+\frac{r^{2} \dot{\beta}^{2}}{2}+\frac{\dot{l}^{2}}{8}+\frac{l^{2} \dot{\phi}^{2}}{8}+\frac{1}{2 r_{1}}+\frac{1}{2 r_{2}}-\frac{\omega^{2}}{8}\left(l-l_{0}\right)^{2},
$$

where $r_{i}=\left(r^{2}+l^{2} / 4+(-1)^{i+1} r l \cos (\phi-\beta)\right)^{1 / 2}, i=1,2$, is the distance of the $i$ th component of the secondary to the primary and $\omega$ is the vibrational frequency of the spring. After including dissipation proportional to $i$ the equations of motion are

$$
\begin{aligned}
\ddot{r} & =r \dot{\beta}^{2}-\frac{1}{2} r R_{+}-\frac{1}{4} l R_{-} \cos (\phi-\beta), \\
\ddot{\beta} & =-2 \frac{\dot{r} \dot{\beta}}{r}-\frac{1}{4} \frac{l}{r} R_{-} \sin (\phi-\beta), \\
\ddot{l} & =l \dot{\phi}^{2}-\omega^{2}\left(l-l_{0}\right)-2 \gamma \dot{l}-\frac{1}{2} l R_{+}-r R_{-} \cos (\phi-\beta), \\
\ddot{\phi} & =-2 \frac{i \dot{\phi}}{l}+\frac{r}{l} R_{-} \sin (\phi-\beta),
\end{aligned}
$$

where $\gamma$ is the dissipation constant and the shorthand notation

$$
R_{ \pm}=\frac{1}{r_{1}^{3}} \pm \frac{1}{r_{2}^{3}}
$$


has been used.

The set of generalized coordinates can be split into two parts. The first subsystem is related to the orbital motion $(r, \beta)$ and the second subsystem describes the internal dynamics (rotation, vibration) of the secondary $(l, \phi)$.

In order to understand the temporal evolution of the full system we can study the energy and angular momentum transfer between the degrees of freedom and subsystems. The angular momenta of the first and second subsystems are defined as

$$
N_{1}=r^{2} \dot{\beta} \text { and } N_{2}=\frac{1}{4} l^{2} \dot{\phi}
$$

respectively. The angular momentum is transferred between the two subsystems but the sum is conserved as we only have central forces $\left(N_{1}+N_{2}=N=\right.$ const.). Because $l \ll r$, in practice $N_{1} \approx N=$ const. The total energy $(E)$ is split into a centre-of-mass energy $E_{c}$ (energy of the first subsystem), a vibrational energy $E_{v i b r}$ and a rotational energy $E_{\text {rot }}$ :

$$
\begin{aligned}
E_{c} & =\frac{1}{2}\left(\dot{r}^{2}+(r \dot{\beta})^{2}\right)-\frac{1}{r}, \\
E_{v i b r} & =\frac{1}{8}\left(\dot{l}^{2}+\omega^{2}\left(l-l_{0}\right)^{2}\right), \\
E_{r o t} & =\frac{1}{8} l^{2} \dot{\phi}^{2}-\frac{1}{2}\left(\frac{1}{r_{1}}+\frac{1}{r_{2}}\right)+\frac{1}{r} .
\end{aligned}
$$

With the above equations we can monitor how the energy is transferred from the centre-ofmass orbit to vibration and rotation and how the total energy decreases through dissipation. We also define the energy of the secondary as

$$
E_{\text {sec }}=E_{\text {vibr }}+E_{\text {rot }} .
$$

The instantaneous angular momentum and the energy transfer are given by the time derivatives of these quantities $(\dot{E}, \dot{N})$.

In order to follow the orbital elements in continuous time, we recall some formulae from the Keplerian problem. The instantaneous dimensionless semi-major axis and the eccentricity can be expressed as

$$
a(t)=-\frac{1}{2 E_{c}(t)}, \quad \varepsilon(t)=\sqrt{1+2 E_{c}(t) N_{1}^{2}(t)}=\sqrt{1-\frac{N_{1}^{2}(t)}{a(t)}} .
$$

respectively. According to (12) the change of $E_{c}$ plays an important role in the change of the semi-major axis and eccentricity. The angle $w$ between the vector pointing to the centre of mass of the secondary and the semi-major axis of the orbit can be obtained from the Keplerian form $r=p /(1-\varepsilon \cos w)$ as

$$
w(t)=\arccos \frac{1-N_{1}^{2}(t) / r(t)}{\varepsilon(t)}
$$

because $p=N_{1}^{2}$. So the angle of apapsis - see Fig. 1(b) - is

$$
\beta_{\text {apapsis }}(t)=\beta(t)-w(t) \text {. }
$$

In this article $t_{i}$ denote the instants when the secondary is at the apapsis (apapsis passages). If a quantity $(f)$ is followed over such a long time that the change of the orbital 
elements is significant, we plot the quantity only at the instants $t_{i}$. In such cases we use the notation $f\left(t_{i}\right)$. To study the long term behaviour of $f$, we use the time average of the time derivative of $f$ over a suitable time period. The choice of the suitable time period depends on the state of the secondary. Generally, it is one period $(q=1)$ but if the system is in a $p: q$ resonance, the average is taken over $q$ periods.

$$
<\dot{f}>_{q}=\frac{1}{t_{i+q}-t_{i}} \int_{t_{i}}^{t_{i+q}} \dot{f}(t) d t .
$$

\section{Resonant and chaotic states}

In dissipative cases $(\gamma>0)$ there is only one attractor, the $1: 1$ resonance, and a circular orbit [7]. The approach towards the attractor typically occurs through a series of resonances. Because of the change of orbital elements we introduce the following resonance functions

$$
\rho(i) \equiv R\left(t_{i}\right)=1+\frac{1}{2 \pi} \int_{t_{i}}^{t_{i+1}} \dot{\alpha} \mathrm{d} t, \quad i=1,2,3 \ldots
$$

The integral term yields the overall rotational angle of the secondary between times $t_{i}$ and $t_{i+1}$ relative to the $r$ axis in units of $2 \pi$; see Fig. 1(a). The unit term express the fact that the radius has carried out approximately one rotation. Because of the small apapsis precession, $R$ or $\rho$ differ slightly from the entire rotational angle of the secondary (in units of $2 \pi$ ). The resonance function is thus a characteristic of the rotation after eliminating the apapsis precession. If the system is in a $p: q$ resonance, function $\rho$ is periodic in $i$, its period is $q$ and

$$
\sum_{i=1}^{q} \rho(i)=p .
$$

The reason for the definition of the functions $R$ is that they are more convenient to plot than $\rho(i)$. In the presence of constant apapsis precession, $R$ would be periodic. Because of the time dependence of the orbital elements in our problem, $R$ is not strictly periodic but is almost so. In a $p: q$ resonance, during $q$ orbital periods the rotation of the secondary relative to the $r$ axis is $p-q$ and the average of the relative rotation is $(p-q) / q$. According to Eq. (16) the relative rotation during one period is $R-1$. Generally, the relative rotation is not exactly equal to the average of the relative rotation but almost so: $R-1 \approx(p-q) / q$. The average of $R$ is therefore $p / q$ and it typically takes on $q$ different values around the average.

To understand better the features of $R$, consider two examples. In a 3:2 resonance, during two orbital periods the relative rotation of the secondary is one. During one of the periods the relative rotation is a little smaller than $1 / 2$ and during the other it is a little larger than $1 / 2$. Thus in the first case $R<0.5+1=1.5$ and in the second case $R>1.5$. When plotting $R$ versus $t_{i}$, if the points are quite dense, we can see two lines near to the value 1.5. The other example is a 2:2 resonant state (see right side of Fig. 2(a) and the mid part of Fig. 2(b)), which is different from 1:1 resonance. In the former during two orbital periods the relative rotation of the secondary is two. During one of the periods the relative rotation is a little less than 0 (negative) and during the other the relative rotation is a little greater than 0 (positive). Thus in the first period $R<1$ and in the second $R>1$. In a 1:1 resonance $R=1$. Although the average of $R$ is the same in both cases $(\bar{R}=1)$, in a 1:1 resonance the position and the 

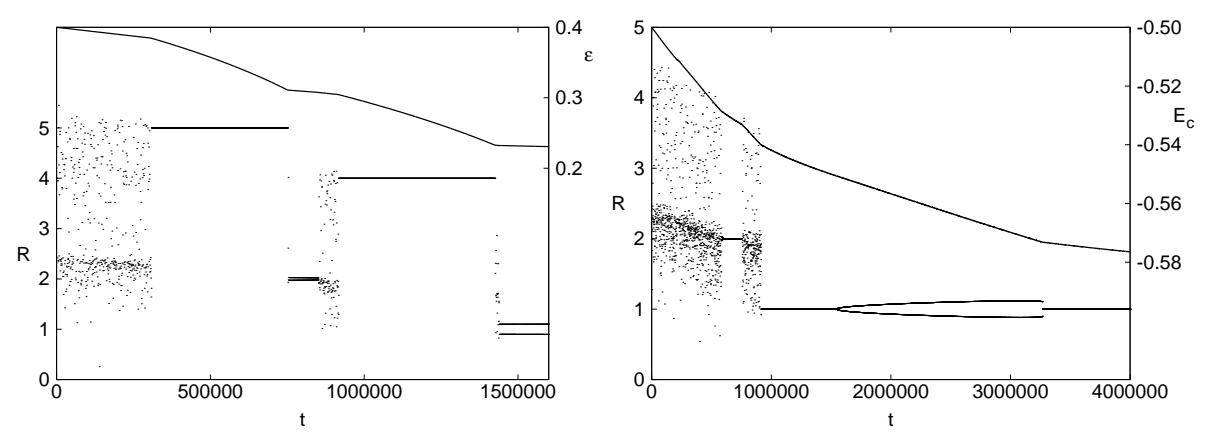

Fig. 2 (a) Resonance function $R\left(t_{i}\right), \varepsilon\left(t_{i}\right)$ (upper continuous line). Parameters and initial conditions: $\omega=10$, $\gamma=1, l_{0}=10^{-3} ; \varepsilon_{0}=0.4, \phi(0)=0, \dot{\phi}(0)=5$. The series of resonances is 5:1, 4:2, 4:1, 2:2. (b) Resonance function $R\left(t_{i}\right), E_{c}\left(t_{i}\right)$ (upper continuous line). Parameters and initial conditions: $\omega=10, \gamma=5, l_{0}=10^{-3}$; $\varepsilon_{0}=0.4, \phi(0)=0, \dot{\phi}(0)=5$. The series of resonances is $2: 1,1: 1,2: 2,1: 1$.

angular velocity relative to the $r$ axis are always the same at apapsis. The 1:1 resonant state and the 2:2 should thus be distinguished from each other because they have quite different features; for example, the amplitude of the libration is larger and the changes of orbital elements are faster in a 2:2 resonance. In this article the term $p: q$ resonant state will be used for resonances different from $1: 1$ only.

Fig. 2(a) shows an example of the time-dependent resonance function where we also plot the temporal change of eccentricity. It is easy to separate the different regimes in the figure. During the first regime $(t=0-300000)$ the rotation of the secondary is chaotic. The orbital motion drives the secondary. Depending on the orbital elements the driving can cause chaotic rotation. The orbital elements are changing and the type of rotation caused by the driving is also changing. By $t \approx 300000$ the driving cannot cause chaotic rotation and the chaotic regime ends.

The second regime is a resonance. If the system is in a $p: q$ resonance the temporal behaviour of the secondary is periodic, thus the average of its energy and the dissipation are constants: $\left\langle\dot{E}_{\text {sec }}\right\rangle_{q}=0$ and

$$
<\dot{E}>_{q}=\frac{1}{t_{i+q}-t_{i}} \int_{t_{i}}^{t_{i+q}}\left(-\frac{1}{2} \gamma i^{2}\right) d t=C_{p: q}=\text { const }<0 .
$$

A constant energy of the secondary can only be maintained if the system pumps the dissipated energy from the orbit to the secondary which means

$$
<\dot{E}_{c}>_{q}=<\dot{E}>_{q}-<\dot{E}_{s e c}>_{q}=C_{p: q}
$$

This is an energy condition for the existence of a $p: q$ resonant state. The subscript $p: q$ expresses the fact that the value of $C$ is determined mainly by the type of resonant state. Of course $C$ also depends on the parameters - e.g., on $l_{0}$ - and on the orbital elements. The resonant state ends - because of the change of orbital elements - when the system cannot pump enough energy to the secondary. If the energy condition (19) is not valid, the resonant state loses its stability.

The third regime (near $t \approx 750000$ ) is quite short. At the beginning of this regime a couple of stable resonant states are present. According to our simulations they behave as 


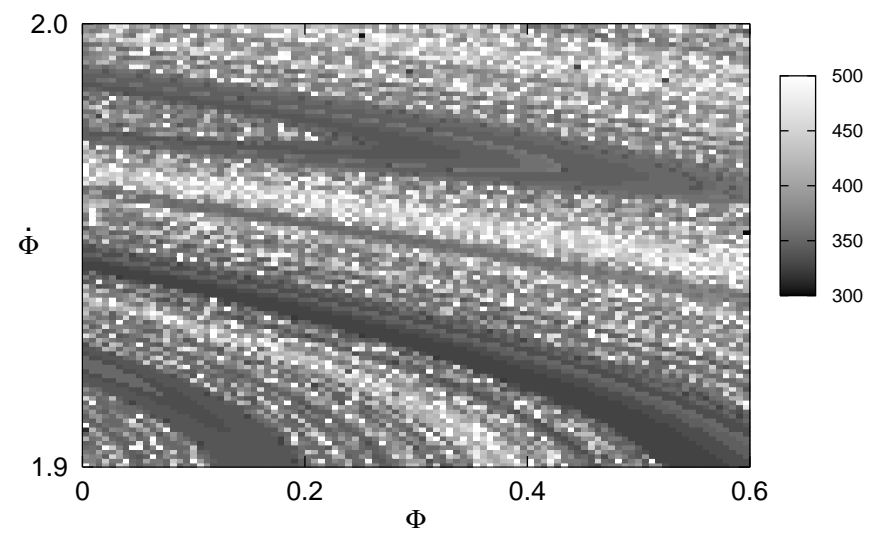

Fig. 3 Lifetime distribution over a piece of the $\phi, \dot{\phi}$ plane with $i=0$. The initial value of $l$ is chosen as $l=l_{0} /\left(\omega^{2}-\dot{\phi}^{2}\right)$. Parameters are $\omega=10, \gamma=10, l_{0}=10^{-4}, \varepsilon=0.1$ (The secondary is at the apapsis). Lifetimes are given in units of orbital periods. The filamentary structure is a manifestation of transient chaos.

quasi-attractors with fractal basin boundaries. If the state of the secondary is close to a boundary between the basins of quasi-attractors, the orbit can exhibit long-time chaos.

The fourth regime is also a resonance. This time the mechanism of the disappearance of the resonance is not the same as in the second regime. The simple quasi-attractor changes into a strange (chaotic) quasi-attractor as a consequence of the change of the orbital elements.

Since all quasi-attractors are simple resonances, any type of chaos present in such dissipative systems can only be of transient type. As is known from the theory of transient chaos [19], [23] the chaotic set is in such cases a non-attracting set, called a chaotic saddle. Orbits initiated close to the stable manifold of the saddle converge towards the chaotic set, stay a long time around it, and will be led eventually to a quasi-attractor along the unstable manifold of the saddle. The presence of a chaotic saddle can be demonstrated by plotting a lifetime distribution over the set of initial conditions. This is done in Fig. 3 where a clear fractal filamentation of the longlived regions can be seen. Although this set of points is similar in appearance to a chaotic attractor, which is an unstable manifold, we emphasize that what we see here is the stable manifold of a chaotic saddle. We have to distinguish two types of transient chaotic regimes. The first (second) type ends without (with) a change of the orbital elements. In Fig. 2(a) the second and fourth chaotic regimes (the short ones) are of the first type and the first and the third chaotic regimes (the longer ones) are of the second type.

The typical behaviour of the eccentricity is a decrease toward zero. In every regime but the $1: 1$ resonance the function of eccentricity is piece-wise concave (see Appendix 8.1). The semi-major axis also decreases and approaches a given value, $a_{\infty}$, determined by the initial conditions (see Appendix 8.2). The further evolution of the system in this case is that it falls into $1: 1$ resonance around $t \approx 1.1 \times 10^{7}$, and the eccentricity tends to zero exponentially as the orbit circularizes. Fig. 2(b) shows another example illustrating that the $1: 1$ resonance can lose stability if the eccentricity is not so small. A similar loss of stability 


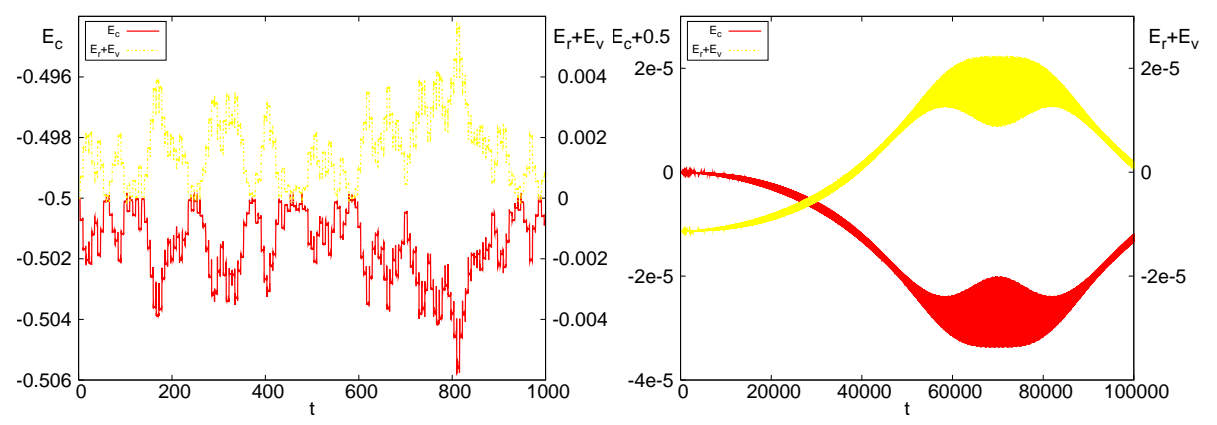

Fig. 4 (a) Energy transfer from the orbit (centre-of-mass kinetic energy, dark, red) to the secondary (rotation and vibration, grey, yellow). After some orbital periods the energy is transferred back to the centre-of-mass motion. $\varepsilon_{0}=0.50, \gamma=0.0, l_{0}=1.0^{-2}, \omega=10$. (b) Longer runs show that this behaviour is periodic. $\varepsilon_{0}=$ $0.30, \gamma=0.0, l_{0}=1.0^{-2}, \omega=10$.
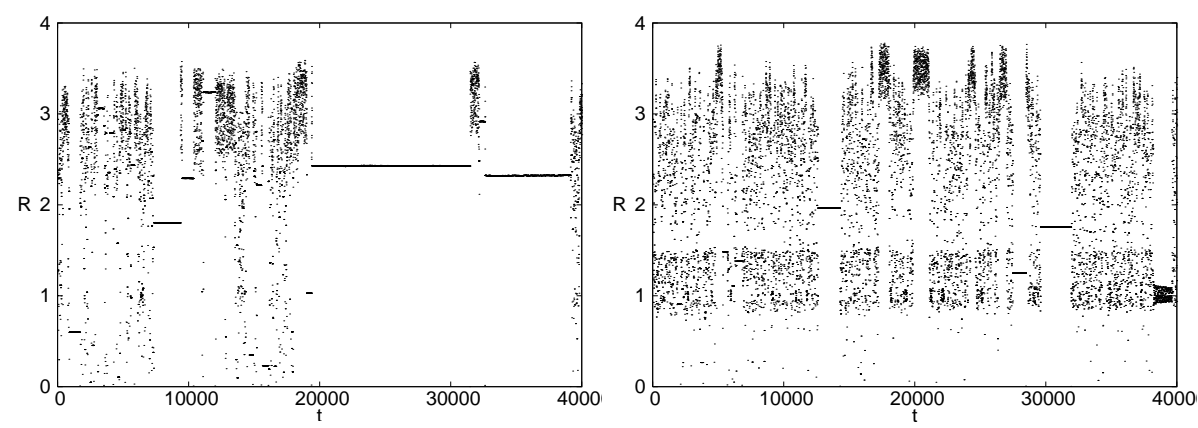

Fig. 5 Function $R(t)$ for elastic secondary with no dissipation, with (a) $\omega=5$ and (b) $\omega=10$. Weaker springs show more resonant states and these remain stable for a longer time.

can be found in a recent paper [22]. Although the authors study a conservative model, a loss of stability of the 1:1 resonance has been found due to the decrease of the eccentricity. In that model the dynamics of the secondary is three-dimensional and the point masses of the secondary are unequal, but because the orbital elements do not change, the loss of stability is not the result of a temporal evolution as in our case. The figure also shows the centre-ofmass energy $E_{c}$; our numerical experience is that the change of $E_{c}$ is exponential only in a 1:1 resonance [7]. In other resonant states the temporal change in $E_{c}$ is approximately linear (for an explanation see Eq. (19)). Another important feature illustrated in Fig. 2(b) is that the decrease of the centre-of-mass energy is, in general, more intense in higher resonances than in $1: 1$ resonance.

In conservative cases $(\gamma=0)$, we do not have any attractor, but the system can still synchronize in several metastable resonances. The spring alone is enough for the secondary to interact with its orbit and temporarily store energy in the form of rotation and vibration. Eventually the energy will go back to the orbit and the system will return close to its initial state. In Fig. 4(a) we show how the energy is transferred from orbit to rotation and back. Stronger springs (higher $\omega$ ) behave more like rigid bodies and hence produce fewer resonances. Weaker springs show a wide variety of synchronizations that can last from a few orbital periods to thousands of orbital periods. A very elastic secondary can easily synchronize in rarer resonances. For instance, we can have resonances with rotation velocity slower 
than the orbital velocity, or retrograde rotation: with rotation in the opposite sense to the orbit. Fig. 5(b) compares the varying resonances that are produced with different springs using $R(t)$ defined in Eq. (16). We can note how a more elastic secondary is more often synchronized and its resonances remain stable for longer times.

\section{Approximate equations of motion}

To calculate the approximate equations of motion we Taylor expand the Lagrangian up to 4 th order in $l /(2 r)$ :

$$
\begin{gathered}
\mathscr{L}=\frac{1}{2}\left[\dot{r}^{2}+r^{2} \dot{\beta}^{2}+\frac{\dot{l}^{2}}{4}+\frac{l^{2}}{4} \dot{\phi}^{2}\right]-\frac{1}{8} \omega^{2}\left(l-l_{0}\right)^{2} \\
+\frac{1}{r}\left[1+\left(\frac{l}{2 r}\right)^{2} P_{2}(\cos \alpha)+\left(\frac{l}{2 r}\right)^{4} P_{4}(\cos \alpha)\right] .
\end{gathered}
$$

The Taylor-expanded equations of motion can be written as $\ddot{\mathbf{q}}=\ddot{\mathbf{q}}^{(0)}+\ddot{\mathbf{q}}^{(1)}+\ddot{\mathbf{q}}^{(2)}+\ldots$ where $\ddot{\mathbf{q}}=(\ddot{r}, \ddot{\beta}, \ddot{l}, \ddot{\phi})$ and

$$
\begin{gathered}
\ddot{\mathbf{q}}^{(0)}=\left(\begin{array}{c}
r \dot{\beta}^{2}-\frac{1}{r^{2}} \\
-2 \frac{\dot{r} \dot{\beta}}{r} \\
l \dot{\phi}^{2}-\omega^{2}\left(l-l_{0}\right)-2 \gamma \dot{l} \\
-2 \frac{i \dot{\phi}}{l}
\end{array}\right), \\
\ddot{\mathbf{q}}^{(1)}=\left(\begin{array}{c}
0 \\
0 \\
-\frac{l}{r^{3}}\left(1-3 \cos ^{2} \alpha\right) \\
-\frac{3}{2} \frac{1}{r^{3}} \sin 2 \alpha
\end{array}\right), \quad \ddot{\mathbf{q}}^{(2)}=\left(\begin{array}{c}
\frac{3}{8} \frac{l^{2}}{r^{4}}\left(1-3 \cos ^{2} \alpha\right) \\
\frac{3}{8} \frac{l^{2}}{r^{5}} \sin 2 \alpha \\
0 \\
0
\end{array}\right), \ldots
\end{gathered}
$$

Another form of the Taylor-expanded equations of motion is

$$
\begin{array}{c|c|c}
\text { 0th order } & \text { 1st order } & 2 \text { order } \\
\ddot{r}=r \dot{\beta}^{2}-\frac{1}{r^{2}} & +\frac{3}{8} \frac{l^{2}}{r^{4}}\left(1-3 \cos ^{2} \alpha\right) \\
\ddot{\beta}=-2 \frac{\dot{r} \dot{\beta}}{r} & +\frac{3}{8} \frac{l^{2}}{r^{5}} \sin 2 \alpha \\
\ddot{l}=l \dot{\phi}^{2}-\omega^{2}\left(l-l_{0}\right)-2 \gamma \dot{l} & -\frac{l}{r^{3}}\left(1-3 \cos ^{2} \alpha\right) \\
\ddot{\phi}=-2 \frac{3}{l} & -\frac{3}{2} \frac{1}{r^{3}} \sin 2 \alpha &
\end{array}
$$

In general, the $n$th order approximation contains the $n$th power of $l / r$ in the equations of $\ddot{r}, r \ddot{\beta}, \ddot{l}$ and $l \ddot{\phi}$ (accelerations). 
In the 0th order approximation $\left(\ddot{\mathbf{q}}=\ddot{\mathbf{q}}^{(0)}\right)$ the system of equations splits into two parts. The first pair of equations $(\ddot{r}, \ddot{\beta})$ forms the equations of motion of the pure Keplerian problem and the second pair $(\ddot{l}, \ddot{\phi})$ describes the problem of two rotating point masses connected by a frictional spring.

It is worth emphasizing that in the 1st-order approximation $\left(\ddot{\mathbf{q}}=\ddot{\mathbf{q}}^{(0)}+\ddot{\mathbf{q}}^{(1)}\right)$ the secondary has no feedback on the centre-of-mass motion at all. That is why the first order approximation has important qualitative differences from the exact model: (i) The orbital elements are not changing; there is no circularization. (ii) It is a driven nonlinear system in which the drive is not changing in time. Depending on the orbital elements the attractor(s) can be simple or strange ones. Of course in the case of a strange attractor the dynamics of the secondary is permanently chaotic and in the case of simple attractors the dynamics can be either regular or transiently chaotic depending on the initial conditions. (iii) The orbit pumps energy into the secondary but the orbital energy remains constant. (iv) The total angular momentum is not constant. (v) The dimension of the phase space of this problem is only 5 . In spite of these differences, it is important to consider this approximation because a couple of its features are reflected in the exact model.

We can also define approximations 1a and 2a. Approximation 1a is the 1st-order approximation without the term $q_{3}^{(1)}=-l / r^{3}\left(1-3 \cos ^{2} \alpha\right)$, i.e., an equation corresponding to rigid-body rotation of the secondary, provided $l=$ const. In this approximation the action of the primary on the spring is neglected. Approximation $2 \mathrm{a}$ is the 2nd-order approximation without the term $q_{2}^{(2)}=3 / 8 l^{2} / r^{5} \sin 2 \alpha$ in the equation of $\ddot{\beta}$. The term kept in the equation of $\ddot{r}$ describes a central force perturbation proportional to $1 / r^{4}$ provided $l$ and $\alpha$ are constant. The force is then $3 / 8 l^{2} / r^{4}\left(1-3 \cos ^{2} \alpha\right)$ and an analytic expression (Eq. (32)) exists for the apapsis precession.

In general, the trajectories calculated by the approximate and exact equations deviate from each other because of the chaoticity of the dynamics; in such cases we expect only the statistical behaviour to be the same. On the other hand, if the motion is regular - as in a resonance - then the approximate equations are expected to be rather accurate.

The Taylor-expanded equations give us a facility to understand the response of the secondary to the changes of orbital elements. By using the second-order approximate equations

$$
\dot{E}_{c}=\dot{r}\left(\ddot{r}+\frac{1}{r^{2}}\right)+r \dot{r} \dot{\beta}^{2}+r^{2} \dot{\beta} \ddot{\beta}=\dot{r} q_{1}^{(2)}+r^{2} \dot{\beta} q_{2}^{(2)}
$$

and the time average of the change of the centre-of-mass energy can be written as

$$
\begin{array}{r}
<\dot{E}_{c}>_{q}=\frac{1}{t_{i+q}-t_{i}} \int_{t_{i}}^{t_{i+q}}\left(\dot{r} q_{1}^{(2)}+r^{2} \dot{\beta} q_{2}^{(2)}\right) d t= \\
\frac{1}{t_{i+q}-t_{i}} \frac{3}{8} \int_{t_{i}}^{t_{i+q}}\left(\frac{\dot{r}}{r^{4}}\left[l^{2}\left(1-3 \cos ^{2} \alpha\right)\right]+\frac{\dot{\beta}}{r^{3}}\left[l^{2} \sin 2 \alpha\right]\right) d t
\end{array}
$$

We use for illustrative purposes a $5: 2$ resonance and the initial eccentricity is 0.1 . Fig. 6(a) [(b)] shows the first (the fraction) and the second (expression in square bracket) factors of the first [second] term in the integral over two orbital periods at different eccentricities. The amplitudes of the two first factors are directly proportional to the eccentricity because the eccentricity and the change of the semi-major axis are relatively small. Because of the energy condition, the integral has to be constant. Therefore function $\alpha(t)$ has to change. 


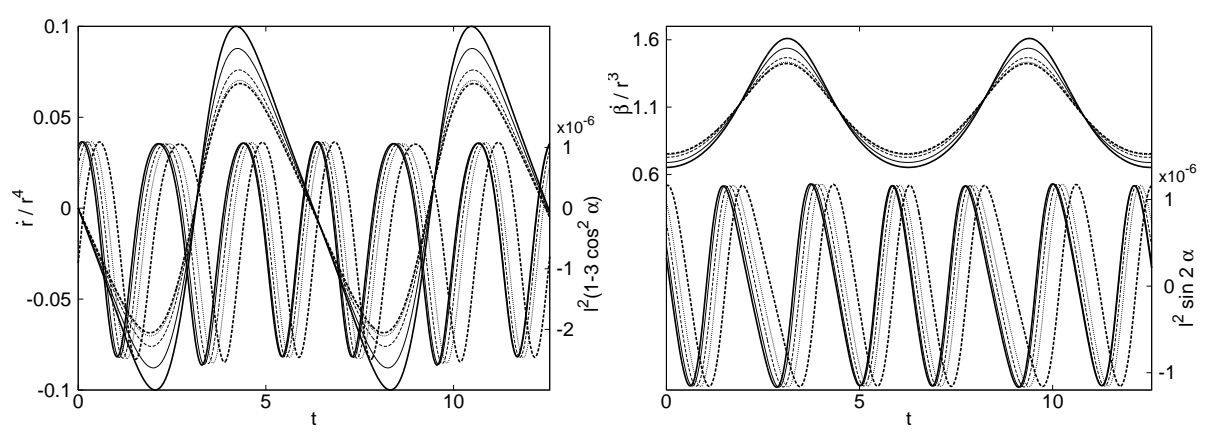

Fig. 6 (a) Temporal change of the factors of the first term in the integral (23) over two orbital periods at different eccentricities. The secondary is at apapsis at $t=0$. The line styles correspond to the eccentricities: 0.9 (heavy continuous), 0.8 (thin continuous), 0.7 (thin dashed), 0.65 (thin dotted) and 0.63 (heavy dashed). (b) Temporal change of the factors of the second term in the integral (23).

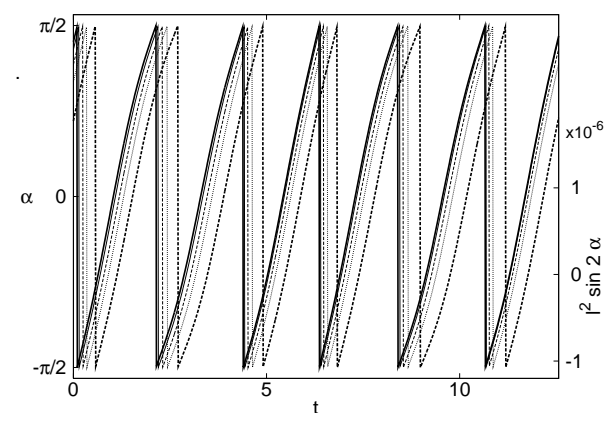

Fig. 7 The shift of function $\alpha(t)$ with eccentricity. The line styles mark the eccentricity values as in Fig. 6.

Fig. 7 shows the function $\alpha(t)$ at $\varepsilon=0.9,0.8,0.7,0.65$, and 0.63 . As the figure displays, the response of the secondary is a shift of $\alpha(t)$. The shift becomes faster as the end of the resonance is approaching.

\section{Temporal evolution of the orbital elements}

In order to obtain a continuous-time estimate of the orbital elements we use equations for

$$
\begin{aligned}
& \dot{\varepsilon}=\frac{1}{\varepsilon}\left(\dot{E}_{c} N_{1}^{2}+2 E_{c} N_{1} \dot{N}_{1}\right), \\
& \dot{a}=\frac{1}{2} \frac{1}{E_{c}^{2}} \dot{E}_{c}=2 a^{2} \dot{E}_{c} .
\end{aligned}
$$

These equations appear in several models which treat the secondary as a spatially extended object, see e.g. [24]. The point is that $\dot{E}_{c}$ and $\dot{N}_{1}$ cannot be expressed by $\varepsilon$ and $a$ only, and the set of equations is thus not closed. Special approximate formulas for $\dot{E}_{c}$ and $\dot{N}_{1}$ are typically needed obtain closure. We are not forced to do so since the numerical values of these quantities easily follow from (2)-(5) and (7), (8). In fact we consider (24), (25) only as diagnostic equations investigated along with the numerical solution of our model. 
For later purposes we also write down an equation for $\beta_{\text {apapsis }}$, used in the same diagnostic sense. By taking the time derivative of (14), (13) and using (7) and (12) we find:

$$
\dot{\beta}_{\text {apapsis }}=\dot{\beta}+\frac{\varepsilon\left(3 r^{2} \dot{r} \dot{\beta}^{2}+2 r^{3} \dot{\beta} \ddot{\beta}\right)+\dot{\varepsilon}\left(1-r^{3} \dot{\beta}^{2}\right)}{\dot{r} \varepsilon \sqrt{a\left(1-\varepsilon^{2}\right)}} .
$$

The leading-order behaviour of these quantities follows from the second-order approximate equations. Substituting

$$
\dot{N}_{1}=2 r \dot{r} \dot{\beta}+r^{2} \ddot{\beta} \approx r^{2} q_{2}^{(2)},
$$

and Eq. (22) into (24) and (25), we obtain

$$
\begin{gathered}
\dot{\varepsilon}=\frac{1}{\varepsilon}\left[\left(\dot{r} q_{1}^{(2)}+r \dot{\beta}^{2} q_{2}^{(2)}\right) N_{1}^{2}+2 E_{c} N_{1} r^{2} q_{2}^{(2)}\right], \\
\dot{a}=2 a^{2}\left(\dot{r} q_{1}^{(2)}+r^{2} \dot{\beta} q_{2}^{(2)}\right) .
\end{gathered}
$$

In a similar way, one finds from (26)

$$
\dot{\beta}_{\text {apapsis }}=\frac{2 r^{3} \dot{\beta} \varepsilon q_{2}^{(2)}-\left(r^{3} \dot{\beta}^{2}-1\right) \dot{\varepsilon}}{\dot{r} \varepsilon \sqrt{a\left(1-\varepsilon^{2}\right)}} .
$$

\subsection{Apapsis precession}

The apapsis precession $\Delta \beta$ is obtained by multiplying $\dot{\beta}_{\text {apapsis }}$ by the instantaneous period $T(t)=2 \pi a(t)^{3 / 2}$ :

$$
\Delta \beta(t)=2 \pi \sqrt{a^{3}(t)} \dot{\beta}_{\text {apapsis }}(t)
$$

This equation provides the instantaneous tendency for producing a given apapsis precession. This can, in certain phases of the motion, be of opposite sign than after a full period, i.e., than the average of $\Delta \beta(t)$ over a period. A well-known result [12] for $\Delta \beta$ in the presence of a dimensionless perturbative force of the type $-\kappa / r^{4}, \kappa \ll 1$ yields

$$
\Delta \beta_{\text {pert }}(t)=2 \pi \frac{\kappa(t)}{N_{1}^{4}(t)}
$$

where $\kappa(t)=\frac{3}{8} l^{2}(t)\left(1-3 \cos ^{2} \alpha(t)\right)$, under the assumption that $\kappa(t)$ changes slowly.

In a time independent naive approximation when $\kappa(t)$ is considered to be constant with $\alpha \approx 0$ and $l=l_{0}, N_{1}=N$

$$
\Delta \beta_{\text {naive }}=\frac{3 \pi}{2} \frac{l_{0}^{2}}{N^{4}} .
$$

In order to test the accuracy of the naive and the perturbative approximations of the apapsis precession we compare the numerical results in the case of the simplest, 1:1 resonance. Fig. 8 (a) shows $\Delta \beta_{\text {naive }}$ from Eq. (33), the measured $\Delta \beta$ (measurement has been made after a period but the value is represented by a continuous line), $\Delta \beta_{\text {pert }}(t)$ from Eq. (32), its average, and the time average of $\Delta \beta(t)$ from Eq. (31) all evaluated in approximation 2a. Our conclusion is that the naive and the perturbative approximations can only estimate the magnitude of the apapsis precession; they are not suitable to give the exact value. The 

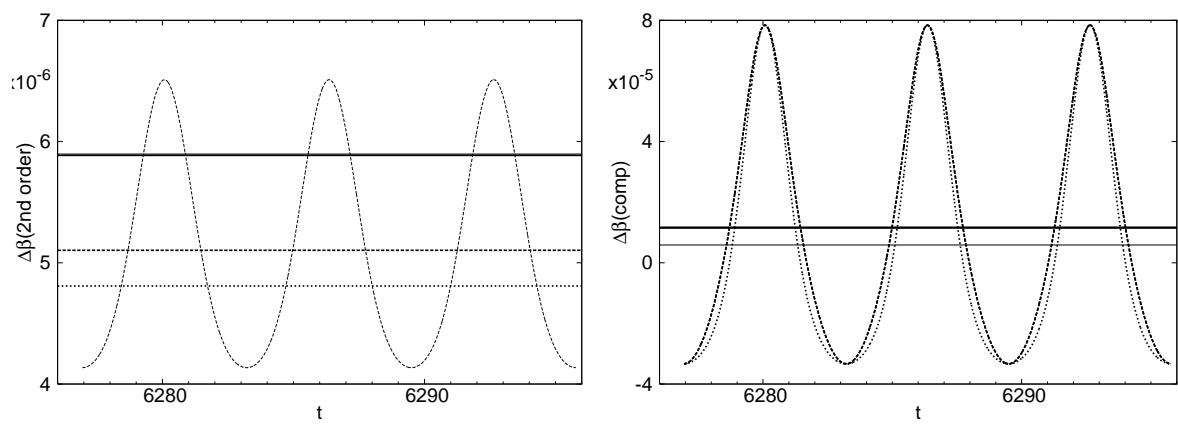

Fig. 8 (a) Comparison of the different methods for the calculation of the apapsis precession in the case of a 1:1 resonance in approximation 2a: numerically measured $\Delta \beta$ (heavy continuous), average of $\Delta \beta(t)$ from Eq. (31) (thin continuous, running very close to the heavy line), $\Delta \beta_{\text {pert }}(t)$ from Eq. (32) (thin dashed, oscillating), average of $\Delta \beta_{\text {pert }}(t)$ (heavy dashed), $\Delta \beta_{\text {naive }}$ from Eq. (33) (heavy dotted). Parameters and initial conditions: $\omega=10, \gamma=1, l_{0}=10^{-3}, \varepsilon_{0}=0.1, \phi(0)=0, \dot{\phi}(0)=5$. (b) Comparison of the apapsis precession obtained by means of the different approximate equations. The measured exact and the exact $\Delta \beta(t)$ averaged coincide with the measured $\Delta \beta$ in 2 nd order (heavy continuous line). The exact $\Delta \beta(t)$ coincides with 2 nd order $\Delta \beta(t)$ (heavy dashed line). Parameters and initial conditions: $\omega=10, \gamma=1, l_{0}=10^{-3}, \varepsilon_{0}=0.1$, $\phi(0)=0, \dot{\phi}(0)=5$.

value of the time-averaged $\Delta \beta(t)$ is practically the same as that of the measured $\Delta \beta$. The difference between the averages of $\Delta \beta(t)$ and $\Delta \beta_{\text {pert }}(t)$ may be because $\kappa$ is also oscillating with an amplitude which is about one fifth of its average value. Therefore, the perturbative result based on the slow variation of $\kappa$ is an educated guess only.

Fig. 8(b) shows that the apapsis precession, Eq. (31), evaluated with the 2nd order approximation and the exact equations coincide (heavy continuous line). This means that the equations of the 2 nd order approximation are sufficient for calculating the apapsis precession to high accuracy. The figure also shows the function $\Delta \beta(t)$ - exact and 2 nd order approximation (heavy dashed), 2a order approximation (heavy dotted) - and the numerically measured apapsis precession in approximation $2 \mathrm{a}$ (thin continuous line). The result is about one half of the measured apapsis precession of the 2nd order approximation and of the exact simulation. This implies that the contribution of the perturbation $3 / 8 l^{2} / r^{4} \sin 2 \alpha$ in the equation of $\ddot{\beta}$ to the apapsis precession is practically as important as the well known central force contribution, Eq. (32), of approximation 2a.

Here we have concentrated on the case of the 1:1 resonance on time scales when the system is still far away from the circularized attractor. Since the interesting time dependencies all die out in the long-time limit, the approximate equations are expected to work even better for later times. The behaviour of the approximation scheme around other resonances is also similar. Altogether, the 2nd-order approximate equations seem to provide a rather accurate description of the entire dynamics, provided that the resonant state under investigation is the same as that predicted by the exact equation. The observation that the approximate equations lead in some cases to different resonances or 'transient attractors' on intermediate time scales appears to be a consequence of the fact that the dynamics between resonances is chaotic and the errors attributable to using a perturbation expansion become drastically amplified.

We can study the influence that the different parameters $-\gamma, \omega, l_{0}, \varepsilon$ - have on the apapsis precession $\Delta \beta_{\text {apapsis. }}$. Looking at Eq. (30), we can see that $\Delta \beta_{\text {apapsis }}$ does not depend on $\gamma$ directly. Numerical results indicate that an indirect dependence cannot be found either for 

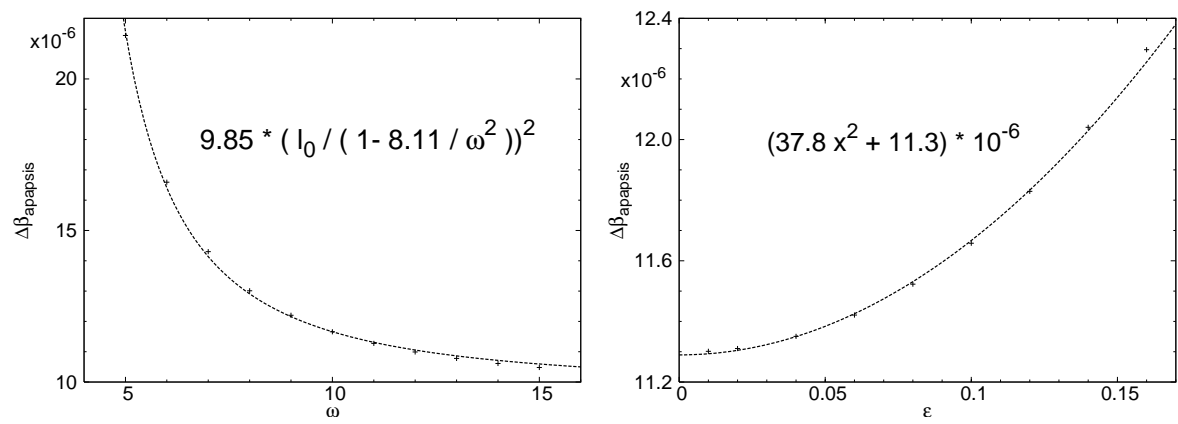

Fig. 9 Numerical data (dots) and fits (continuous lines) for the apapsis precession as a function of spring frequency (a) and eccentricity (b). (a) Parameters are $\gamma=1, l_{0}=10^{-3} ; \varepsilon_{0}=0.05$, and the fitted parameters of (34) are $c_{1}=9.85, c_{2}=8.11$. (b) Parameters are $\omega=10, \gamma=1, l_{0}=10^{-3}$. The form of the fitted function is $a \varepsilon^{2}+b$. The result of the fit is $a=3.78 \cdot 10^{-5}, b=1.13 \cdot 10^{-5}$
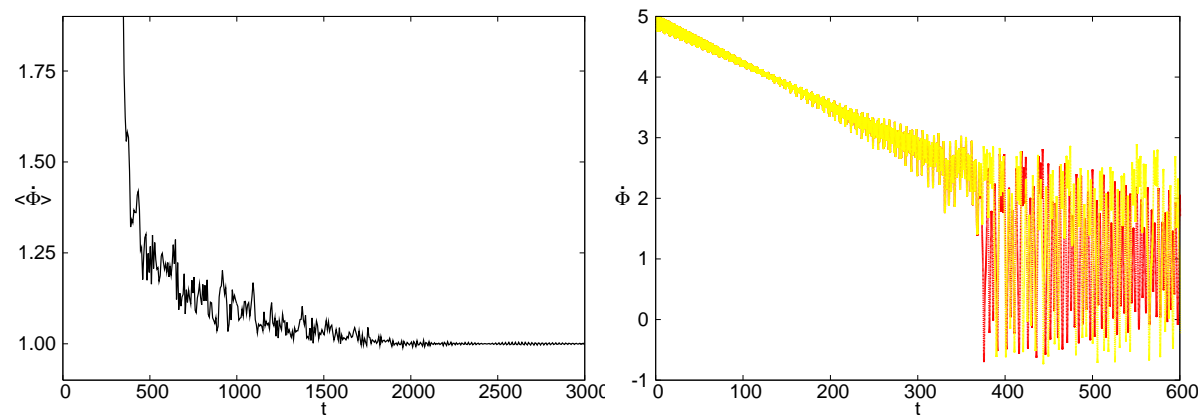

Fig. 10 (a) Average angular velocity with the exact equations. Initial conditions: $\varepsilon_{0}=0.1$ and $\left(\phi_{0}, \dot{\phi}_{0}\right)$ is an element of the grid determined by the values $\phi_{0} \in\{0,1,2,3,4,5,6\}, \dot{\phi}_{0} \in\{4.98,4.99,5.0,5.01,5.02\}$. We used this set of initial conditions for every averaged plot in this article. (b) $\dot{\phi}(t)$ in the 2 th order approximation (dark, red) and with the exact (grey, yellow) equations of motion. Parameters and initial conditions: $\omega=10$, $\gamma=1, l_{0}=10^{-3} ; \varepsilon_{0}=0.1, \phi(0)=0, \dot{\phi}(0)=5$.

a wide range of $\gamma(\gamma<2)$. $\dot{\beta}_{\text {apapsis }}$ (30) depends on $q_{1}^{(2)}$ (through $\dot{\varepsilon}$ ) and $q_{2}^{(2)}$, and the $l$ dependence of both terms is quadratic, so $\dot{\beta}_{\text {apapsis }} \sim l^{2}$. Using the approximation for $l$ (Eq. (43) of the Appendix 8.3) we expect that the $l_{0}$ and $\omega$ dependencies can be written as

$$
\Delta \beta_{\text {apapsis }}=c_{1}\left(\frac{l_{0}}{1-c_{2} / \omega^{2}}\right)^{2}
$$

where $c_{1}$ and $c_{2}$ are fitting parameters. Numerical fits confirm this conjecture. The $l_{0^{-}}$ dependence is perfectly quadratic and Fig. 9(a) shows that expression (34) as a function of $\omega$ is perfectly consistent with the measured data. Fig. 9(b) shows that the eccentricity dependence is basically also quadratic, but as $\varepsilon \rightarrow 0$, the apapsis precession seems to converge to a nonzero value.

\section{Comparison of approximations in chaotic cases}

To produce Fig. 10(a), the exact equations of motion have been integrated with 35 different initial conditions and the time averages of the angular velocity have been calculated in every 

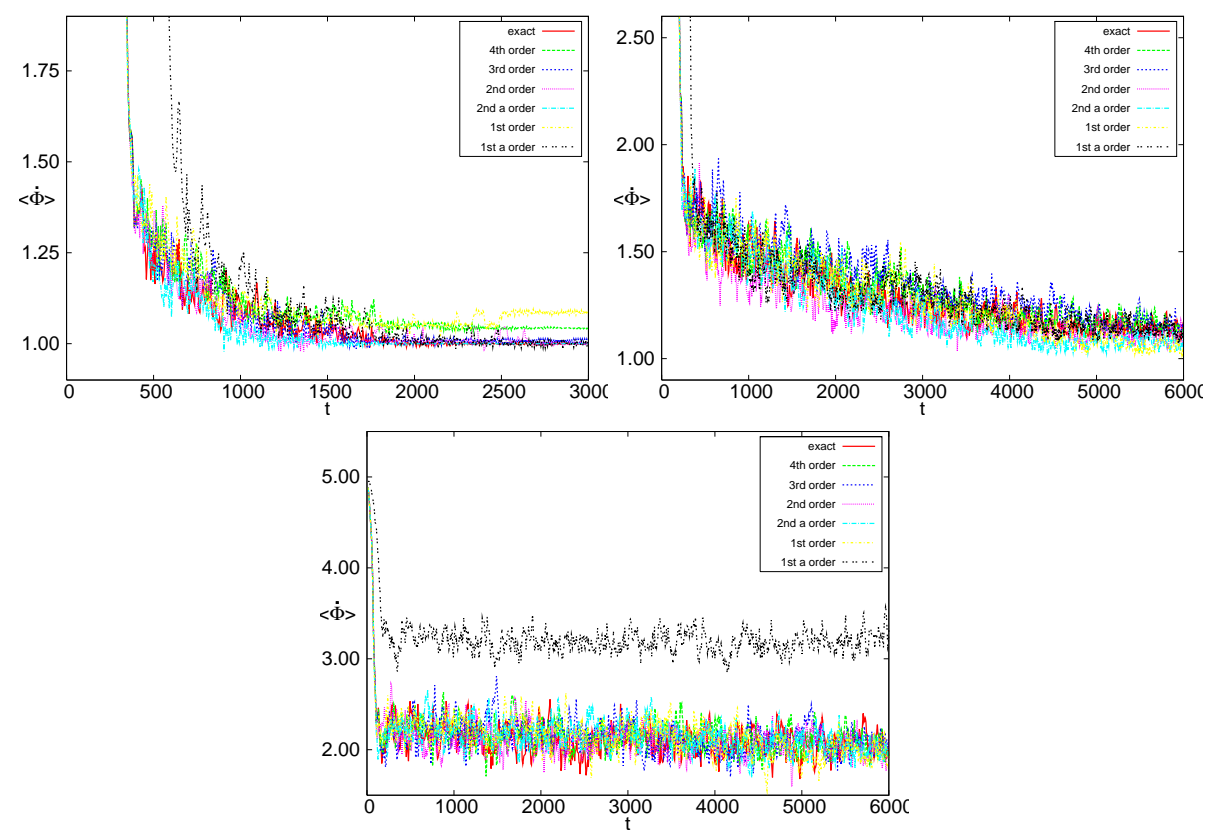

Fig. 11 Comparison of the average behaviour of the different approximate equations. Initial eccentricity is (a) $\varepsilon_{0}=0.1$; (b) $\varepsilon_{0}=0.2$; (c) $\varepsilon_{0}=0.3$.

period. The initial eccentricities of all 35 integrations were the same $(\varepsilon=0.1)$. Fig. 10(a) shows the average of the angular velocities versus time. There is a plateau at the end of the curve, since all 35 cases are in resonance. The height of the plateau is 1 implying that all the cases are in a 1:1 resonance. We integrated the exact and the 2nd-order equations of motion with the same initial conditions. Angular velocity $\dot{\phi}$ versus time can be seen in Fig. 10(b). Here and even in the case of the highest-order approximation the trajectories deviate from each other in the transient chaotic regime $(t>300)$. Based on a study of a couple of similar cases we conclude that the 2nd-order (and higher-order) approximations have the same characteristic features as the exact equations of motion.

We have also studied the statistical behaviour of the different approximations. The same type of plot as Fig. 10(a) has been made to all orders of the approximations in Fig. 11(a). We conclude that all approximations other than 1a exhibit the same statistical behaviour as the exact case. From the graphs the characteristic time, $\tau_{1}$, needed to reach a 1:1 resonance can be estimated. This state is not yet the circularized attractor since $\varepsilon \approx \varepsilon_{0}$ after such short period of time. It takes typically a much longer time — denoted by $\tau$ in [7] — for the attractor, where $\varepsilon=0$, to be reached; in this example $\tau_{1} \approx 2000$ and $\tau \approx 10^{6}$. The plateau of the green, 4th-order curve is slightly higher than 1 . The reason is that one of the 4 thorder cases is in a 5:2 resonance and not in 1:1. Among the 1st-order simulations there were two in a 5:2 resonance, which is why the yellow curve is higher than the green one. It is hardly observable but the dark blue, 3rd-order curve is also higher than 1; this is owing to the presence of a 5:4 resonance. Fig. 11(b) and (c) show a comparison of the exact and the approximate cases with different initial eccentricities. Based on these, we conclude that the higher the eccentricity the greater the time $\tau_{1}$. In Fig. 11(c) the asymptotic value is not 1 , 

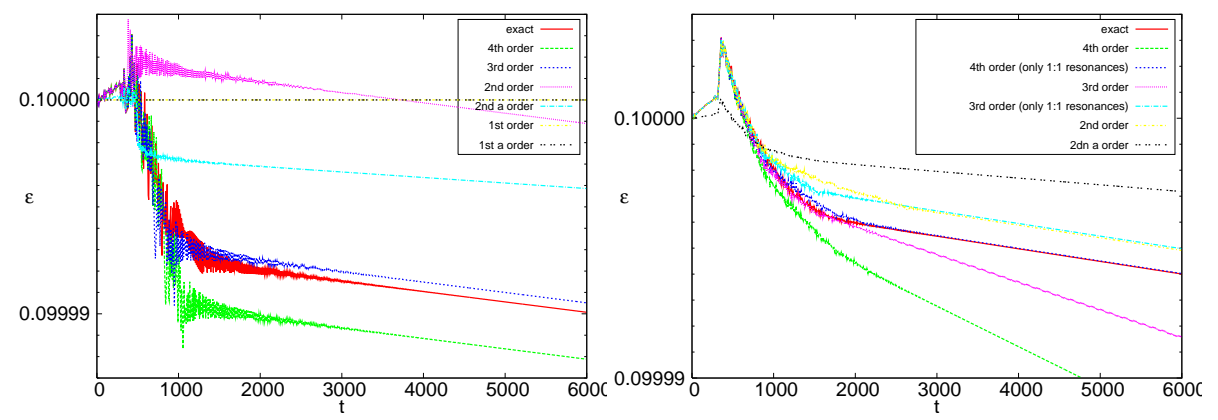

Fig. 12 (a) Comparison of eccentricities with the different approximate equations. Parameters and initial conditions: $\omega=10, \gamma=1, l_{0}=10^{-3} ; \varepsilon_{0}=0.1, \phi(0)=0, \dot{\phi}(0)=5$. (b) Comparison of the averaged eccentricities with the different approximate equations. Parameters and initial conditions: $\omega=10, \gamma=1, l_{0}=10^{-3}$; $\varepsilon_{0}=0.1$.

which suggests that a 1:1 resonance has not yet been reached by the end of the integration; the curve is not smooth because most of the trajectories are not in resonance.

Fig. 12 shows the time dependence of the eccentricity $\varepsilon(t)$ in the different approximate equations. In all cases a 1:1 resonance has been reached by $t \approx 3000$. In the first order approximation there is no feedback of the secondary on the Keplerian problem, therefore $\varepsilon(t)=\varepsilon_{0}=$ const . The asymptotic slopes of the other curves show the rate of decrease of eccentricity. These rates are the same in all cases with the exception of approximation $2 \mathrm{a}$ which indicates that the contribution of the term $3 / 8 l^{2} / r^{5} \sin 2 \alpha$ missing from the equation for $\ddot{\beta}$ is important.

Fig. 12(b) shows the eccentricity averaged over 35 different initial conditions in the different approximate equations. It is a general observation that in resonances different from 1:1 the eccentricity decays much faster. This is the reason why in cases in which there are also other resonances than 1:1 in the ensemble (the 3rd and 4th order) the averaged eccentricities decrease faster. If we average only the 1:1 resonance cases than we obtain the light and the dark blue curves. As expected, the curves of the 2nd and 3rd order are close to each other, and close to the curves of the 4th order and to the exact numerical solution which practically coincide. Fig. 12(b) indicates that in exceptional cases even the 4th-order approximation can lead to a resonance - a 'transient attractor' — different from the exact one. The good agreement between the approximate and exact equations only holds under the condition that the same resonance has been reached.

\section{Discussion}

Spin-orbit resonances between a large primary body and a smaller secondary body are generally studied using models designed for quantitative analysis of a specific instance or a particular part of the problem $[14,9,8,20,18,1,3,5,2,6,22]$. The complexity of these models tends to obscure the fundamental physical principles involved in this interaction. We have instead taken a qualitative dynamical-systems approach, using a minimalistic model that takes into account only the essential ingredients of tidal deformation and dissipation in the secondary body. Despite its simplicity, the model can account for both synchronization into the 1:1 spin-orbit resonance and the circularization of the orbit as the only true asymptotic attractors. Apart from its applicability to tidal synchronization and orbit circu- 
larization of celestial bodies, our model is also relevant to the problem of the dynamics of tethered satellites, which has recently provoked much interest and a number of space missions $[13,22]$.

One interesting open question is whether a pseudo-energy — or energy-like — approach would provide new insight into the problem. In this case we have completely defined the gravitational and mechanical energies - the centre of mass plus rotation and vibration of the secondary - and thus the dissipated energy can be obtained simply by subtraction from the initial energy; the same is true for the evolution between two arbitrary states. As the dissipation is strictly passive the total energy is necessary a decaying function, reaching a constant value only in a situation in which the dissipation term vanishes along the trajectory. In the approach of Sarasola et al. [21], energy dissipation is associated with a local change in phase space volume and on this basis they define a partial differential equation that an energy-like function must satisfy. The same depends on a part of the decomposition of the vector velocity field; the divergence free or rotational part in contrast with the part that carries the whole divergence. Once $H$ is obtained we can calculate the energy derivative along a trajectory of the system which is due to the part carrying the divergence of the vector field. Probably this is the unique new information that can be obtained in our case: an analytical expression instead of the indirect calculation by subtraction of the mechanical plus gravitational energy. Nonetheless the pseudo-energy approach is of interest and we intend to pursue it in future work.

The main conclusions that may be drawn from the present investigations are: The 1:1 resonance is the only stable attractor. Dissipation drives the system towards this synchronization and circularizes the orbit. All other resonances are metastable, or transient attractors, and dissipate energy at a faster pace, and the approach towards the circular orbit is also faster.

If we look to our solar system, we find that all large moons are locked in 1:1 spinorbit resonance with their planets. Of the planets, however, only Mercury is locked into spin-orbit resonance with the Sun, and this is a 3:2 spin-orbit resonance [3]. The presence or absence of locking can be explained in our model through the strength of the coupling [7], which is largest for the moons, and smaller for the planets, of which Mercury has the strongest coupling. Within our minimal model, in which 1:1 is the only asymptotic attractor, such a 3:2 resonance is metastable, and Mercury would eventually decay into 1:1 spin-orbit resonance. On the other hand, it may be possible to stabilize 3:2 resonance in our model by adding to it interactions with further bodies (the other planets); three-dimensionality (noncoplanarity of spin and orbit); and post-Newtonian effects.

Isolating the dominant interactions between the degrees of freedom in terms of a Taylor expansion allows us to to study them separately. In general, the second-order approximation is sufficient to reproduce the typical behaviour of the complete model, as long as we are in the 1:1 resonance. This has been studied for apapsis precession, chaotic regimes and eccentricity evolution.

Finally, we summarize features of our model which are expected to remain valid in more general cases as well. Even if the expression for $\langle\dot{E}\rangle_{q}$ can, in general, be quite complex, the energy condition (19) for resonant states holds in any case. The mechanism of the response of the secondary to the change of the orbital elements should remain similar to in our model. Of course, we have to use the general formula (23) where $q_{1}^{(2)}$ and $q_{2}^{(2)}$ can be rather complicated. 
Acknowledgements We acknowledge projects OTKA NK72037 (Hungary), Hielocris (Spain), CGL200806245-CO2 (Spain), and the Hispano-Hungarian Binational project TeT ESP-34/2006.

\section{References}

1. Celletti, A., Chierchia, L.: Hamiltonian stability of spin-orbit resonances in celestial mechanics. Celest. Mech. Dyn. Astron. 76, 229-240 (2000)

2. Celletti, A., Froeschle, C., Lega, E.: Dynamics of the conservative and dissipative spinorbit problem. Planet Space Sci. 55, 889-899 (2007)

3. Correia, A.C.M., Laskar, J.: Mercury's capture into the $3 / 2$ spin-orbit resonance as a result of its chaotic dynamics. Nature 429, 848-850 (2004)

4. Darwin, G.H.: Tidal Friction and Cosmogony. Cambridge University Press (1908)

5. Dobbs-Dixon, I., Lin, D.N.C., Mardling, R.A.: Spin-orbit evolution of short-period planets. Astrophys. J. 610, 464-476 (2004)

6. Efroimsky, M., Williams, J.G.: Tidal torques: a critical review of some techniques. Celest. Mech. Dyn. Astron. 104, 257-289 (2009)

7. Escribano, B., Vanyo, J., Tuval, I., Cartwright, J.H.E., González, D.L., Piro, O., Tél, T.: Dynamics of tidal synchronization and orbit circularization of celestial bodies. Phys. Rev. E 78, 036216 (2008)

8. Gladman, B., Quinn, D.D., Nicholson, P., Rand, R.: Synchronous locking of tidally evolving satellites. Icarus 122, 166-192 (1996)

9. Goldreich, P., Soter, S.: Q in the solar system. Icarus 5, 375-389 (1966)

10. Hurford, T.A., Greenberg, R.: Tidal evolution by elongated primaries: Implications for the Ida/Dactyl system. Geophys. Res. Letters 27, 1595-1598 (2000)

11. Hut, P.: Tidal evolution in close binary systems. Astron. Astrophys. 99, 126-140 (1981)

12. Landau, L.D., Lifshitz, E.M.: Course of Theoretical Physics - Mechanics, vol 1. Pergamon Press (1981)

13. Levin, E.M.: Dynamic Analysis of Space Tether Missions. Advances in the Astronautical Sciences, vol. 126. AAS Publication, San Diego (2007)

14. MacDonald, G.J.F.: Tidal friction. Rev. Geophys. 2, 467-541 (1964)

15. Mardling, R.A.: The role of chaos in the circularization of tidal capture binaries. I. The chaos boundary. Astrophys. J. 450, 722-731 (1995)

16. Mardling, R.A.: The role of chaos in the circularization of tidal capture binaries. II. Long-time evolution. Astrophys. J. 450, 732-747 (1995)

17. Munk, W.H., MacDonald, G.J.F.: The Rotation of the Earth: A Geophysical Discussion. Cambridge University Press (1975)

18. Murray, C.D., Dermott, S.F.: Solar System Dynamics. Cambridge University Press (1999)

19. Ott, E.: Chaos in Dynamical Systems. Cambridge University Press (2002)

20. Rasio, F.A., Tout, C.A., Lubow, S.H., Livio, M.: Tidal decay of close planetary orbits. Astrophys. J. 470, 1187-1191 (1996)

21. Sarasola, C., d'Anjou, A., Torrealdea, F.J., Moujahid, A.: Energy-like functions for some dissipative chaotic systems. Int. J. Bifurcation and Chaos 15, 2507-2521 (2005)

22. Sidorenko, V.V., Celletti, A.: A Spring-mass model of tethered satellite systems: properties of planar periodic motions. Celest. Mech. Dyn. Astron. 107, 209-230 (2010)

23. Tél, T., Gruiz, M.: Chaotic Dynamics, An introduction based on classical mechanics. Cambridge University Press (2006) 
24. Witte, M., Savonije, G.J. Orbital evolution by dynamical tides in the solar type stars. Astron. Astrophys. 386, 222-236 (2002) 


\section{Appendix}

8.1 Temporal change of the eccentricity

As we saw there are two typical temporal behaviours of $E_{c}$; linear and exponential. From Eq. (12) and considering that the approximate equation $N_{1} \approx N$ is valid we can estimate the temporal change of eccentricity.

a. Exponential temporal behaviour of $E_{c}$ Assuming the function of $E_{c}\left(t_{i}\right)$ to be in the form $E_{c}\left(t_{i}\right)=E_{c, \infty}+A_{E c} e^{-\lambda_{E c} t_{i}}$ we obtain an exponential function for the eccentricity

$$
\varepsilon\left(t_{i}\right) \approx \sqrt{1+2\left(E_{c, \infty}+A_{E c} e^{-\lambda_{E c} t_{i}}\right) N^{2}}=\sqrt{2 A_{E c} e^{-\lambda_{E c} t_{i} N^{2}}}
$$

where we used that $\sqrt{1+2 E_{c \infty} N^{2}}=\varepsilon_{\infty}=0$. The eccentricity thus also decays exponentially and its exponent is the half of the exponent of the function $E_{c}\left(t_{i}\right)\left(\lambda_{\varepsilon}=\lambda_{E c} / 2\right)$.

b. Linear temporal behaviour of $E_{c}$ In $p: q$ resonant states the temporal behaviour of $E_{c}$ is linear with the negative slope $C_{p: q}$ so $E_{c}\left(t_{i}\right)=C_{p_{q}} t_{i}+E_{c, 0}$ where $E_{c, 0}=E_{c}\left(t_{0}\right)$. The eccentricity at the instant $t_{0}$ is

$$
\varepsilon_{0}=\varepsilon\left(t_{0}\right)=\sqrt{1+2 E_{c, 0} N^{2}}
$$

Using this we obtain the temporal change of the eccentricity

$$
\varepsilon\left(t_{i}\right)=\sqrt{1+2\left(C_{p: q} t_{i}+E_{c, 0}\right) N^{2}}=\sqrt{\varepsilon_{0}^{2}+2 C_{p: q} N^{2} t}
$$

which is always a concave function.

\subsection{Radius of the circularized orbit}

Consider a circular orbit in a 1:1 resonance: $a_{\infty}=r=$ const, $\dot{\beta}_{\infty}=$ const,$\dot{\phi}_{\infty}=$ const,$l_{\infty}=$ const, $\alpha=0$ and $\dot{l}=0$. The parameters of the circularized orbit are determined from the condition

$$
\dot{\phi}_{\infty}=\dot{\beta}_{\infty}
$$

Because $l_{\infty} \ll a_{\infty}$ from Eq. (12), a good approximation can be obtained for $a_{\infty}$ if we consider that $N_{1} \approx N_{0}$. By writing down Eq. (12) initially we obtain

$$
N_{0}^{2}=1-\varepsilon_{0}^{2}
$$

In the final state $\varepsilon_{\infty}=0,0=1-N_{0}^{2} / a_{\infty}$ and thus

$$
a_{\infty}=1-\varepsilon_{0}^{2}
$$

The larger the initial eccentricity $\left(\varepsilon_{0}\right)$, the smaller the final radius of the orbit $\left(a_{\infty}\right)$. It is important to note that $a_{\infty}$ is independent of any other parameters i.e., $\gamma, \omega$ or $l_{0}$. 


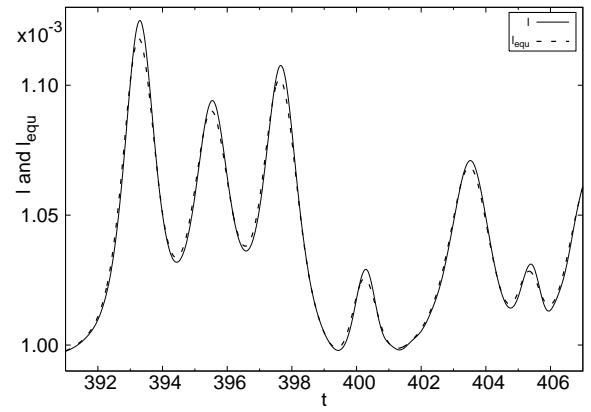

Fig. 13 Comparison of the measured $l$ (continuous line) and $l_{\text {equ }}$ calculated by Eq. (43) (dashed line). Parameters and initial conditions: $\omega=10, \gamma=1, l_{0}=10^{-3} ; \varepsilon_{0}=0.1, \phi(0)=0, \dot{\phi}(0)=5$.

\subsection{Quasistationary behaviour of $l$}

We introduce $\Delta l=l-l_{0}$. Its equation of motion in the 2 nd order approximation is

$$
\Delta \ddot{l}=l \dot{\phi}^{2}-\omega^{2} \Delta l-2 \gamma \dot{\Delta l}-\frac{l}{r^{3}}\left(1-3 \cos ^{2} \alpha\right) .
$$

According to numerical results $\Delta l / l \ll 1$ which is why the left side and the third term of the right side are neglectable. The second term is generally not negligible. After writing $\Delta l=l-l_{0}$ we obtain

$$
0=l \dot{\phi}^{2}-\omega^{2}\left(l-l_{0}\right)-\frac{l}{r^{3}}\left(1-3 \cos ^{2} \alpha\right)
$$

This means that $\ddot{l} \approx 0$, and the spring is almost in equilibrium at each moment. On expanding $l$ we obtain

$$
l_{\text {equ }}=\frac{\omega^{2} l_{0}}{\omega^{2}-\dot{\phi}^{2}+\frac{1}{r^{3}}\left(1-3 \cos ^{2} \alpha\right)}=l_{0} \frac{1}{1-\frac{1}{\omega^{2}}\left[\dot{\phi}^{2}+\frac{1}{r^{3}}\left(3 \cos ^{2} \alpha-1\right)\right]}
$$

where we added the subscript $e q u$. A comparison of $l(t)$ and $l_{e q u}(t)$ is shown in Fig. 13. 